\title{
The Role of Microscopy in Indoor Air Quality Investigations
}

\author{
R.B. Simmons
}

Biological Imaging Core Facility, Dept. of Biology, College of Arts and Sciences, MSC8L0389, 33

Gilmer St., Unit 8, Georgia State University, Atlanta GA 30303-3088

Numerous studies have demonstrated that fungi are capable of colonizing a range of indoor construction and finishing materials as well as a number of air handling system components ${ }^{1,2,3,4,5,6}$, Many of the species found are known to produce mycotoxins and demonstration of the presence of 'toxic mould' in both the workplace and in dwellings has become a major issue. Mould issues now are the focus of litigation or are currently in contention in a number of states and hundreds of millions of dollars are at stake ${ }^{7,8}$.

The indoor environment harbors a variety of microorganisms, including bacteria and fungi. Under normal indoor conditions most of these organisms are present as dormant forms. The fungi persist either as conidia (asexual spores), sexual spores, or hyphal fragments. The presence of water in an environment may lead to the growth and proliferation of fungal species, sometimes resulting in deterioration of the colonized substrates with possible negative effects on IAQ. Fungi growing on various substrates often produce volatile organic compounds that are released into the air. Humans find a number of these substances irritating and may detect them at extremely low concentrations $(<$ 1 part per million). Detection of these offensive substances is often the first indicator of a fungal colonization problem and may lead to an investigation into the source. Methodology of these investigations may be crucial to an accurate assessment of the location and scope of the problem ${ }^{9}$.

Emerging health concerns about fungi fall into four major areas. Adventitious pathogens, such as Aspergillus fumigatus, A. flavus and A. niger are airborne fungi primarily of concern to individuals with suppressed immune systems which make them susceptible to infection. These fungi are commonly found in both the indoor and outdoor environments. Overt fungal pathogens (Blastomyces, Cryptococcus, Coccidiodes and certain dermatophytes) may occasionally be found indoors but rarely colonize indoor substrates. Potentially toxigenic fungi such as Stachybotrys chartarum are currently the focus of much attention. Representatives of this species complex may colonize indoor substrates, particularly gypsum wallboard and ceiling tiles, under certain conditions of moisture and temperature. Review of the literature indicates that there is inadequate evidence linking indoor exposure to fungal toxins with reported symptomology ${ }^{10}$. By far the most common fungi colonizing the indoor environment are Aspergillus, Cladosporium, and Penicillium. Species of these genera have all proven to be allergenic for certain individuals ${ }^{11}$.

Until recently, air sampling techniques (both volumetric sampling and settling plates) have been commonly applied when fungal colonization was suspected. Growth media-based techniques provide some small insight into the nature of a suspected problem, but are inadequate for a complete investigation and are not recommended under the current guidelines. Cryptic colonization of structural materials may produce olfactory evidence of fungal growth without yielding any culture data from air samples. Culture data demonstrates the presence of only those fungi amenable to the culture media applied, and no single culture medium is good for all types of fungi commonly found in the indoor environment. Direct sampling of surfaces and application of light or scanning electron 
microscopy technologies has been the method of our choice to identify the actively colonizing fungi. In conjunction with environmental chamber studies, direct observations facilitate evaluations of remediation processes.

Heightened fear of illness or perception of incapacity from fungal toxins has fueled the increasing frequency of litigation in cases of fungal colonization. Whether there is any factual basis for the role of fungi in such litigations may often be resolved in part by direct microscopic examination of materials and surfaces.

References

1. Ahearn, D.G., et al., J. Ind. Microbiol. 8 (1991) 277-280.

2. Ahearn, D.G., et al., Environments for People: IAQ92 (1992) 101-105.

3. Price, D.L., et al. J. Ind. Microbiol. 13 (1994) 154-158.

4. Ezeonu, I.M. et al., Appl. Environ. Microbiol. 60 (1994) 2149-2151.

5. Simmons and Crow, J. Ind. Microbiol. 14 (1995) 41-45.

6. Simmons, R.B. et al., Am. Indust. Hyg. Assoc. J. 58 (1997) 900-904.

7. Goldstein and Striefsky, HPAC Eng. Sept. (2002) 36-39.

8. Alitz and Hatem, HPAC Eng. Sept. (2002) 72.

9. Ahearn, D.G. et al., J. Ind. Microbiol. 16 (1996) 280-285.

10. Page and Trout, Am. Indust. Hyg. Assoc. J. 62 (2001) 644-648.

11. Summerbell, et al., J. Med. Vet. Mycol. 32 (1994) 277-286.
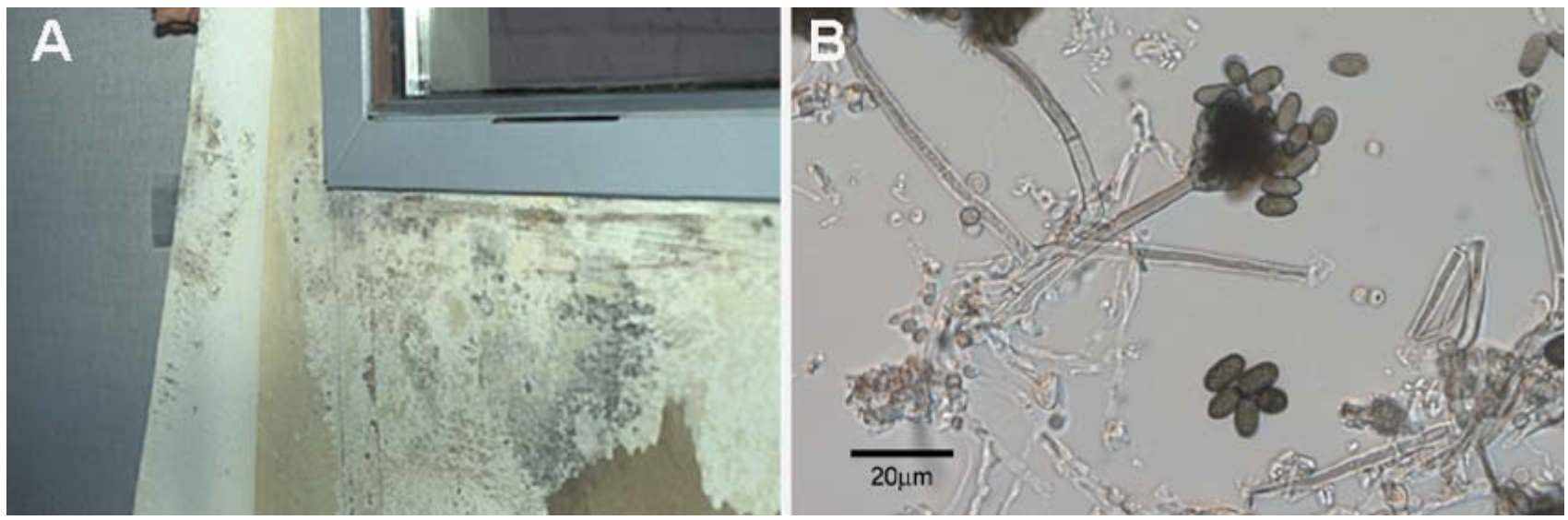

Figure 1. A. Cryptic colonization of gypsum wallboard by a mixed population of fungi. Fungi grew between the wallboard surface and plasticized wall covering material and were not detected by conventional air sampling techniques. B. Stachybotrys, Aspergillus, and Acremonium species observed by direct sampling of colonized construction material. Scale bar $=20 \mu \mathrm{m}$. 